\title{
Visualizing the non-equilibrium dynamics of photoinduced intramolecular electron transfer with femtosecond X-ray pulses
}

\author{
Sophie E. Canton ${ }^{1, \star}$, Kasper S. Kjær ${ }^{2,3, \star}$, György Vankó ${ }^{4}$, Tim B. van Driel ${ }^{3}$, Shin-ichi Adachi ${ }^{5}$, Amélie Bordage ${ }^{4, \dagger}$, \\ Christian Bressler ${ }^{6,7}$, Pavel Chabera ${ }^{8}$, Morten Christensen ${ }^{3}$, Asmus O. Dohn ${ }^{9}$, Andreas Galler 6 , \\ Wojciech Gawelda6 ${ }^{6}$ David Gosztola ${ }^{10}$, Kristoffer Haldrup ${ }^{3}$, Tobias Harlang ${ }^{8}$, Yizhu Liu ${ }^{11}$, Klaus B. Møller ${ }^{9}$, \\ Zoltán Németh ${ }^{4}$, Shunsuke Nozawa ${ }^{5}$, Mátyás Pápai ${ }^{4}$, Tokushi Sato ${ }^{5, \dagger}$, Takahiro Sato ${ }^{12, \dagger}$, \\ Karina Suarez-Alcantara ${ }^{1} \dagger$, Tadashi Togashi ${ }^{13}$, Kensuke Tono ${ }^{13}$, Jens Uhlig ${ }^{8}$, Dimali A. Vithanage ${ }^{8}$, \\ Kenneth Wärnmark ${ }^{11}$, Makina Yabashi ${ }^{12}$, Jianxin Zhang ${ }^{11, \dagger}$, Villy Sundström ${ }^{8}$ \& Martin M. Nielsen ${ }^{3}$
}

Ultrafast photoinduced electron transfer preceding energy equilibration still poses many experimental and conceptual challenges to the optimization of photoconversion since an atomic-scale description has so far been beyond reach. Here we combine femtosecond transient optical absorption spectroscopy with ultrafast $X$-ray emission spectroscopy and diffuse $\mathrm{X}$-ray scattering at the SACLA facility to track the non-equilibrated electronic and structural dynamics within a bimetallic donor-acceptor complex that contains an optically dark centre. Exploiting the 100-fold increase in temporal resolution as compared with storage ring facilities, these measurements constitute the first $X$-ray-based visualization of a non-equilibrated intramolecular electron transfer process over large interatomic distances. Experimental and theoretical results establish that mediation through electronically excited molecular states is a key mechanistic feature. The present study demonstrates the extensive potential of femtosecond X-ray techniques as diagnostics of non-adiabatic electron transfer processes in synthetic and biological systems, and some directions for future studies, are outlined.

\footnotetext{
${ }^{1}$ Department of Synchrotron Radiation Instrumentation, Lund University, PO Box 118, 22100 Lund, Sweden. ${ }^{2}$ Centre for Molecular Movies, Niels Bohr Institute, University of Copenhagen, DK-2100 Copenhagen, Denmark. ${ }^{3}$ Centre for Molecular Movies, Department of Physics, Technical University of Denmark, DK-2800 Lyngby, Denmark. ${ }^{4}$ Wigner Research Centre for Physics, Hungarian Academy Sciences, PO Box 49, H-1525 Budapest, Hungary. ${ }^{5}$ High Energy Accelerator Research Organization, 1-1 Oho, Tsukuba, Ibaraki 305-0801, Japan. ${ }^{6}$ European XFEL, Albert-Einstein Ring 19, D-22761, Hamburg, Germany. ${ }^{7}$ The Hamburg Centre for Ultrafast Imaging, Luruper Chaussee 149, 22761 Hamburg, Germany. ${ }^{8}$ Department of Chemical Physics, Lund University, PO Box 124, S-22100 Lund, Sweden. ${ }^{9}$ Department of Chemistry, Technical University of Denmark, DK-2800, Kongens Lyngby, Denmark. ${ }^{10}$ Center for Nanoscale Materials, Argonne National Laboratory, 9700 South Cass Avenue, Argonne, Illinois 60439, USA. ${ }^{11}$ Department of Chemistry, Centre for Analysis and Synthesis, Lund University, S-22100 Lund, Sweden. ${ }^{12}$ RIKEN SPring-8 Center, 1-1-1 Kouto, Sayo-cho, Sayo-gun, Hyogo 679-5148, Japan. ${ }^{13}$ Japan Synchrotron Radiation Research Institute (JASRI), 1-1-1 Kouto, Sayo-cho, Sayo-gun, Hyogo 679-5198, Japan. * These authors have contributed equally to the work. †Present addresses: Institut de Chimie Moléculaire et des Matériaux d'Orsay, Université Paris-Sud, UMR CNRS 8182, 15 rue Georges Clémenceau, 91405 Orsay Cedex, France (A.B.); Center for Free-Electron Laser Science, Deutsches Elektronen-Synchrotron DESY, Notkestrase 85, 22607 Hamburg, Germany (T.S.); Department of Chemistry, School of Science, The University of Tokyo, 7-3-1 Hongo, Bunkyo-ku, Tokyo 113-0033, Japan (T.S.); Unidad Morelia del Instituto de Investigaciones en Materiales, Universidad Nacional Autónoma de México, Antigua Carretera a Pátzcuaro No. 8701, Col. Ex Hacienda de San José de la Huerta, CP 58190 Morelia, Michoacán, México (K.S.-A.); School of Environmental and Chemical Engineering, Tianjin Polytechnic University, Tianjin 300387, China (J.Z.). Correspondence and requests for materials should be addressed to S.E.C. (email: sophie.canton@desy.de) or to K.S.K. (email: kaspersk@gmail.com) or to M.M.N. (email: mmee@fysik.dtu.dk).
} 
P hotoinduced electron transfer (ET) is one of the primary events associated with the functionality of solvated molecular assemblies, ranging from simple dyads to large proteins. Studies across physics, chemistry and biology have been rationalizing how structure and surroundings are shaping the free-energy landscape for this fundamental process ${ }^{1}$. The respective influence of intra- and intermolecular factors can often be readily disentangled for ET involving equilibrated reactants ${ }^{2}$. This is no longer the case on the ultrafast timescale, since neither the nuclear degrees of freedom nor the first solvation shell has yet adapted to the electronic configuration of the nascent excited state ${ }^{3}$. The photoinduced charge migration is thus proceeding out of equilibrium. The fundamental importance and practical relevance of this regime have both emerged from the novel results obtained with multidimensional optical spectroscopies, which have been enabled by the progress in femtosecond laser technologies ${ }^{4-7}$. The relaxation from the Franck-Condon state to the lowest thermally equilibrated excited state can be generally described in terms of interligand $\mathrm{ET}$, intramolecular vibrational relaxation, internal conversion and intersystem crossing ${ }^{8,9}$. Investigating the interplay between these deactivation pathways in simple mononuclear complexes has been geared intensively towards optimizing the excited-state properties of light emitters and sensitizers, for example, the versatile families of polypyridyl compounds derived from $\left[\mathrm{Ir}^{\mathrm{II}}(\mathrm{ppy})_{3}\right], \quad\left[\mathrm{Ru}^{\mathrm{II}}(\mathrm{bpy})_{3}\right]$ or $\left[\mathrm{Os}^{\mathrm{II}}(\mathrm{bpy})_{3}\right]^{10-12}$. As lightharvesting building blocks, they can be integrated to photomolecular devices that transform solar energy into electrical or chemical potential via ultrafast ET. For such functional complexes, maximizing the yields of long-lived charge-separated species, while minimizing adverse heat dissipation are highly complementary goals pursued to increase their selectivity and stability, hence their overall efficiency. Ultimately, controlling the dynamics of non-equilibrated ET would enable harnessing the largely untapped potential of 'hot' transitions for driving numerous photoconversion schemes with significant excess of stored electronic energy ${ }^{13-15}$

Deciphering the dynamics of intramolecular ET as the system relaxes and thermalizes requires mapping the spatial and temporal redistribution of the energy deposited initially by photoabsorption. So far, this problem has been tackled with the spectroscopic tools developed for monitoring solvation dynamics and vibronic cooling in large molecules ${ }^{16}$. This usually relies on correlating information gathered from measurements conducted separately in different spectral regions with varying experimental conditions (for example, solute concentrations or laser fluences). A salient complication faced in the ultraviolet-visible and near infrared range is the low degree of element- and spin specificity displayed by optical transitions, which have to obey strict dipole selection rules. The applicability of these spectroscopies is thus restricted to participating states that are optically bright. In addition, the dynamics of non-equilibrated ET often exhibit distinctive excitation wavelength dependencies and multi- or non-exponential kinetic behaviours. Although the interpretation can be assisted by density functional theory (DFT), timedependent DFT and molecular dynamics (MD) simulations ${ }^{3}$, the current understanding of the process needs to be refined down to the molecular level before descriptive and predictive models can be firmly validated. The exploration of this conceptual frontier is anticipated to advance rapidly at X-ray free-electron laser (XFEL) facilities, where optical pump-X-ray probe detection schemes can now track the electronic and structural dynamics on the atomic scale with femtosecond resolution in the gas ${ }^{17}$, solution $^{18,19}$ and solid phase $\mathrm{e}^{20-22}$.

Here we combine X-ray Emission Spectroscopy (XES) and $\mathrm{X}$-ray Diffuse Scattering (XDS) with the highly intense ultrashort pulses delivered by the SACLA XFEL facility ${ }^{23}$ to address the long-standing challenge of characterizing non-equilibrated ET in donor-acceptor assemblies that contain optically dark active sites. The bimetallic complex studied in this work consists of a lightharvesting, ruthenium (Ru)-based chromophore linked to an optically dark cobalt (Co) electron sink by a bridge that mediates ultrafast ET. This prototypical dyad exemplifies the wide class of synthetic and natural photocatalysts for which the coupled electronic and structural dynamics are only partially understood beyond the decay of the Franck-Condon state. In the present study, Co $\mathrm{K} \alpha_{1}$ XES based on $2 p-1 s$ electronic transitions is employed to follow the ET from the photoexcited Ru centre to the Co centre with inherent element- and spin sensitivity ${ }^{24-26}$. Analysis of the azimuthally integrated one-dimensional X-ray scattering signals $S(Q)$, as a function of the momentum transfer $Q$ is used to extract the phototriggered structural changes and the kinetics of thermalization ${ }^{27-32}$. Going beyond the limited information delivered by transient optical absorption spectroscopy, these simultaneous XFEL experiments retrieve the timescales that are necessary to fully describe the nonequilibrated ET in the photoexcited dyad. The immediate prospects offered by this general methodology to the diagnostics and optimization of 'hot' photoactive molecular complexes are further highlighted.

\section{Results}

Tracking the optically bright dynamics in photoexcited RuCo. The bimetallic RuCo complex $\left[(\mathrm{bpy}){ }_{2}^{1} \mathrm{Ru}^{\mathrm{II}}(\mathrm{tpphz}){ }^{1} \mathrm{Co}^{\mathrm{III}}(\mathrm{bpy})_{2}\right]^{5+}$ (with bpy = bipyridine, tpphz = tetrapyrido (3,2-a:2 $2^{\prime} \quad 3^{\prime}-c: 3^{\prime \prime}, 2^{\prime \prime}$ $-h:: 2^{\prime \prime \prime}, 3^{\prime \prime \prime}-j$ ) phenazine) $)^{33}$ studied in this work is shown in Fig. 1a. Its $\mathrm{PF}_{6}$ salt was synthesized following an improved protocol ${ }^{34}$. This dyad consists of a ${ }^{1} \mathrm{Ru}^{\text {II }}\left(t_{2 g}\right)^{6}$ centre and a ${ }^{1} \mathrm{Co}^{\text {III }}$ $\left(t_{2 g}\right)^{6}$ centre in the low-spin (LS) state, which are held apart at fixed distance $(\sim 13 \AA)$ and orientation by the rigid bridge tpphz. This planar $\pi$-conjugated system is known to act as a 'molecular wire' capable of mediating ultrafast $\mathrm{ET}^{35}$. The chemical structure is abbreviated as $\left[{ }^{1} \mathrm{Ru}^{\mathrm{II}}={ }^{1} \mathrm{Co}{ }^{\mathrm{III}}(\mathrm{LS})\right]$ hereafter. The steady-state

a

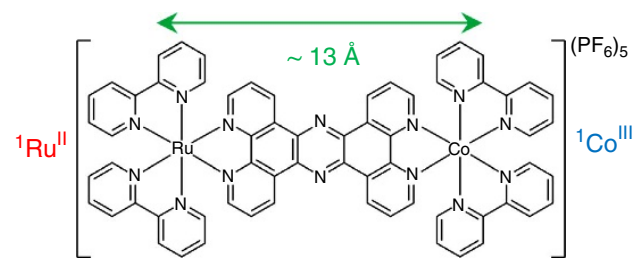

b

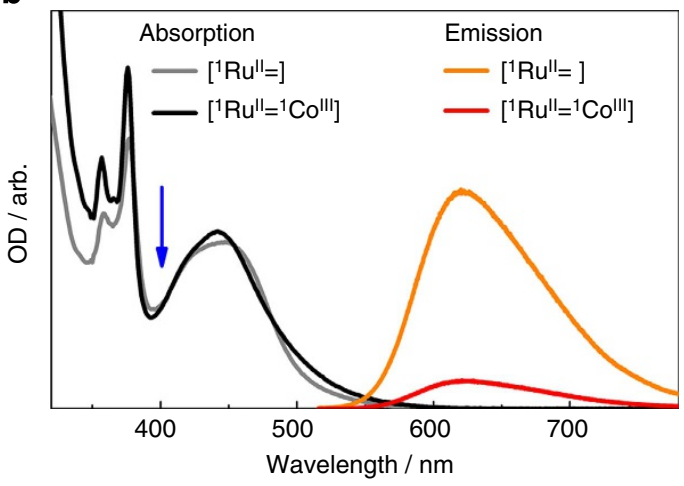

Figure 1 | The $\left[{ }^{\mathbf{1}} \mathbf{R} \mathbf{u}^{\mathbf{I}}={ }^{\mathbf{1}} \mathbf{C o}^{\mathbf{I I I}}\right]$ complex. (a) The molecular structure of the dyad studied in this work. The Ru and Co centres are held $13 \AA$ apart by the tpphz rigid bridge. (b) Absorption and emission spectra of $\left[{ }^{1} \mathrm{Ru}{ }^{11}=\right]$ and $\left[{ }^{1} \mathrm{Ru}^{\prime \prime}={ }^{1} \mathrm{Co} \mathrm{O}^{\mathrm{III}}\right]$ in acetonitrile. The pump wavelength used for all the optical and $\mathrm{X}$-ray experiments is indicated by the blue arrow. 
optical absorption spectrum of the $\left[(\text { bpy }){ }_{2}^{1} \mathrm{Ru}^{\mathrm{II}}(\mathrm{tpphz})\right]^{2+}$ moiety (denoted $\left.\left[{ }^{1} \mathrm{Ru}^{\mathrm{II}}=\right]\right)$ in acetonitrile $(\mathrm{MeCN})$ is displayed in Fig. 1b. The tpphz ligand-centred transitions and the two singlet metal-to-ligand charge transfer (MLCT) transitions $\mathrm{Ru} \rightarrow$ bpy and $\mathrm{Ru} \rightarrow \operatorname{tpphz}$ give rise, respectively, to the absorbance in the $340-380,400-440$ and $440-550 \mathrm{~nm}$ regions ${ }^{36,37}$. The steady-state emission spectrum of $\left[{ }^{1} \mathrm{Ru}^{\mathrm{II}}=\right]$ excited at $400 \mathrm{~nm}$ (Fig. 1b) closely resembles that from the ${ }^{3} \mathrm{MLCT}$ in $\left[\mathrm{Ru}^{\mathrm{II}}(\text { phen })_{3}\right]^{35-37}$ (where phen=phenanthroline) with a maximum intensity at $622 \mathrm{~nm}$. Although the optical absorption spectrum of $\left[{ }^{1} \mathrm{Ru}^{\mathrm{II}}={ }^{1} \mathrm{Co}^{\mathrm{III}}\right.$ (LS) $]$ does not change appreciably after coordination of the ${ }^{1} \mathrm{Co}^{\mathrm{III}}$ moiety, its emission yield is reduced by $>80 \%$ without noticeable alteration in the spectral lineshape. The dynamics of this quenching process can be followed with femtosecond transient optical absorption spectroscopy (TOAS) as shown in Fig. 2a (Supplementary Note 1). On selective photoexcitation of the ${ }^{\mathrm{MLCT}}$ in the Ru chromophore at $400 \mathrm{~nm}$, a band peaking at around $625 \mathrm{~nm}$ appears quasiinstantaneously and decays very rapidly (red trace in Fig. 2b). After $25 \mathrm{ps}$, the transient absorption spectrum has evolved into a broader band of weaker intensity (blue trace in Fig. 2b). A globalfit analysis of the transient absorption signal within the first $30 \mathrm{ps}$ reveals that three Decay-Associated Spectra DAS $1, \mathrm{DAS}_{2}$ and $\mathrm{DAS}_{3}$ (Fig. 2c) are needed to describe adequately the dynamics. The details of this analysis are outlined in the Supplementary Note 1 . The reduced pyrazine state ${ }^{35-37}\left(\mathrm{DAS}_{1}\right)$ decays with a $490 \pm 17 \mathrm{fs}$ lifetime to a hot ${ }^{3} \mathrm{MLCT}\left(\mathrm{DAS}_{2}\right)$ reminiscent of the ${ }^{3} \mathrm{MLCT}$ in $\left[\mathrm{Ru}(\mathrm{phen})_{3}\right]^{2+}$ (refs $\left.35-37\right)$. The cooling to the thermalized excited state occurs with an $8 \pm 3$ ps time constant $\left(\mathrm{DAS}_{3}\right)$. Figure $2 \mathrm{~d}$ shows the kinetics traces at 460,540 and $625 \mathrm{~nm}$. A model accounting for the steady-state and timeresolved spectroscopic observations can be built, where the relaxation of the Franck-Condon state branches out into two parallel intramolecular ET pathways (Supplementary Fig. 1). In the first route, the bridge-localized CT state $\left[(\mathrm{bpy})_{2}^{2} \mathrm{Ru}^{\mathrm{III}}\right.$ $($ tpphz $\left.) \bullet{ }^{1} \mathrm{Co}^{\mathrm{III}}(\mathrm{bpy})_{2}\right]^{5+}\left(\right.$ denoted $\left.\left[{ }^{2} \mathrm{Ru}^{\mathrm{III}}(=\bullet)^{1} \mathrm{Co}^{\mathrm{III}}(\mathrm{LS})\right]\right)$ is populated instantaneously $(<50 \mathrm{fs})$ and decays on the subpicosecond timescale. In the second route, the phen-like portion of the tpphz ligand is formally reduced. This is the state that gives rise to the quenched steady-state emission observed in Fig. 1b. It should be noted that such a model holds when the pump wavelength is varied across the absorption band between 370 and $480 \mathrm{~nm}$ (Supplementary Fig. 2). All the extracted time constants exhibit the pronounced excitation wavelength dependency that could be expected from an intermolecular ET event proceeding out of equilibrium, as mentioned in the introduction. From these TOAS measurements, it is clearly possible to determine the residence time of the electron on the bridge. However, the dynamics that take place at the Co centre completely elude detection. The deceptively simple questions 'when is the electron reaching the ${ }^{1} \mathrm{Co}^{\mathrm{III}}$ centre?', and 'how does the relaxation at the reduced $\mathrm{Co}^{\mathrm{II}}$ centre take place?' remain unanswered. Very similar unresolved issues about the actual electronic localization and phototriggered structural dynamics are encountered in the studies of fully functional photoactive materials (for example, homogeneous or heterogeneous photocatalysts ${ }^{38-41}$ and organic-inorganic hybrid solar cells ${ }^{42,43}$ ) whenever weak optically bright or optically dark transitions play a role in the photoconversion process. This particular $\left[{ }^{1} \mathrm{Ru}^{\mathrm{II}}={ }^{1} \mathrm{Co}{ }^{\mathrm{III}}\right.$ (LS) $]$ compound is therefore a very well-suited candidate to demonstrate the unique capabilities of ultrafast X-ray techniques for investigating such processes. As shown below, femtosecond XES and XDS are successfully employed in this work to capture the optically dark electronic and structural dynamics that take place within the photoexcited donor-bridgeacceptor complex.

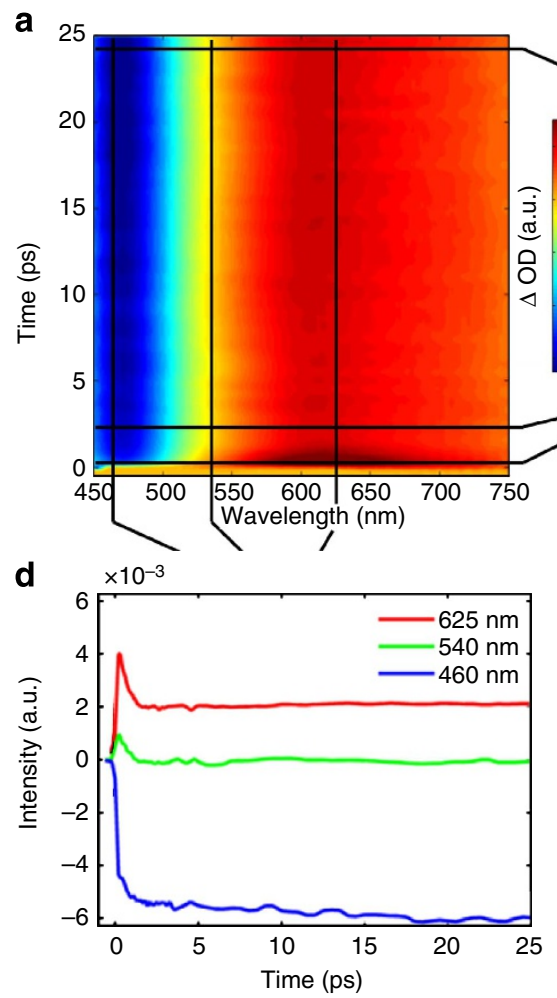

b

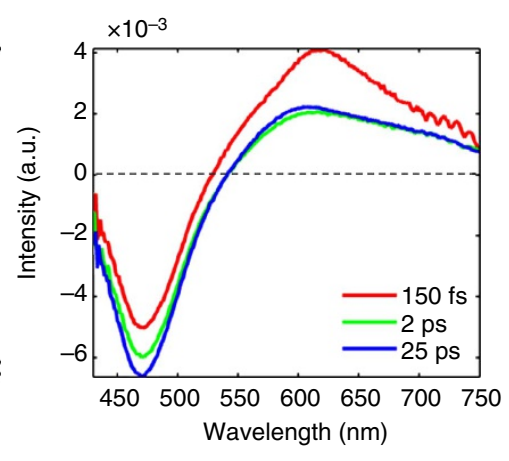

c

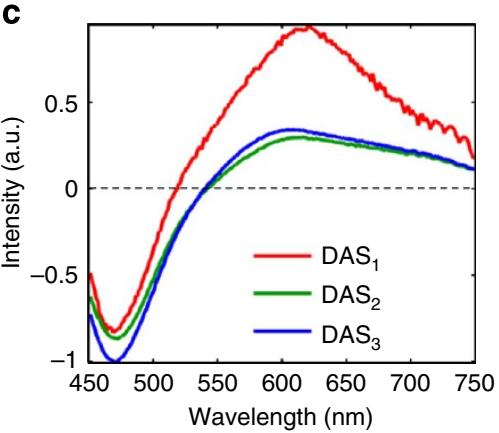

Figure 2 | Ultrafast optical absorption spectroscopy. Clockwise: (a) Transient optical absorption spectra of $\left[{ }^{1} \mathrm{Ru}^{\mathrm{II}}={ }^{1} \mathrm{Co} \mathrm{O}^{\mathrm{III}}\right]$ excited at $400 \mathrm{~nm}$ as a function of pump-probe time delay. (b) Transient absorption spectra at three pump-probe time delays: $150 \mathrm{fs}$, and 2 and 25 ps. (c) The three decay-associated spectra $\mathrm{DAS}_{1}, \mathrm{DAS}_{2}$ and $\mathrm{DAS}_{3}$ returned by the global analysis fitting procedure (GA-fit). (d) Kinetic traces over the first $25 \mathrm{ps}$ at three different probe wavelengths: 460,540 and $625 \mathrm{~nm}$. 
Tracking the optically dark dynamics in photoexcited RuCo. A pump-probe setup accommodating simultaneous XES and XDS measurements ${ }^{44,45}$ was implemented at BL3 of the SACLA XFEL (Fig. 3). Figure $4 \mathrm{a}$ displays the Co $\mathrm{K} \alpha_{1}$ XES difference signal $\Delta S_{\mathrm{XES}}(t)$ [laser ON $_{\mathrm{ON}}(t)-$ laser $_{\mathrm{OFF}}$ ] acquired at time delays $t$ fixed to 2.5 (red trace) and $20 \mathrm{ps}$ (blue trace) after the selective excitation at $400 \mathrm{~nm}$ of the ${ }^{1}$ MLCT state in the Ru ${ }^{\mathrm{II}}$ moiety (Supplementary Note 2; Supplementary Fig. 3). This X-ray emission line originates from the secondary $2 p_{3 / 2} \rightarrow 1 s$ transition subsequent to $1 s$ core ionization. Steady-state $24,26,46$ and time-resolved ${ }^{45,47,48}$ experiments at storage ring facilities have established that for spin-state transitions (SSTs) in $3 d$ transition metal ions, the full width at half maximum (FWHM) of the $\mathrm{K} \alpha_{1}$ lines (hence its inverse maximum intensity) is directly proportional to the number of unpaired electrons. This spectral feature therefore carries information about the total spin momentum of the X-ray absorbing centre. To assign the transient signal $\Delta S_{\mathrm{XES}}(t)$, the spectra were compared with a static reference trace constructed by subtracting the normalized lineshapes measured for a $\left(\pi \mathrm{t}_{2 \mathrm{~g}}\right)^{6}$ $\left[{ }^{1} \mathrm{Co}{ }^{\mathrm{III}}\right]$ centre in the LS state from that of a $\left(\pi \mathrm{t}_{2 \mathrm{~g}}\right)^{5}\left(\sigma \mathrm{e}_{\mathrm{g}}\right)^{2}\left[{ }^{4} \mathrm{Co}^{\mathrm{II}}\right]$ centre in the high-spin (HS) state (Supplementary Note 3; Supplementary Fig. 4). The strong similarity with the $\Delta S_{\mathrm{XES}}$ at $t=20$ ps demonstrates that ET from $\mathrm{Ru}^{\mathrm{II} \star}$ to ${ }^{1} \mathrm{Co}{ }^{\mathrm{III}}$ (LS) and a SST at the reduced $\mathrm{Co}^{\mathrm{II}}$ centre have both taken place during this time interval. Such finding concurs with previous synchrotronbased transient X-ray absorption experiments, where the reduced $\mathrm{Co}^{\mathrm{II}}$ moiety was observed in the quartet HS state ${ }^{34}$ with an 80 ps temporal resolution. Relative scaling of the reference trace (black dashed trace in Fig. 4a) to $\Delta S_{\text {XES }}$ at $t=20$ ps delivers $65 \pm 8 \%$ as the fraction of $\left[{ }^{2} \mathrm{Ru}{ }^{\mathrm{III}}={ }^{4} \mathrm{Co}{ }^{\mathrm{II}}(\mathrm{HS})\right]$. It depends both on the initial concentration of photoexcited $\left[{ }^{1} \mathrm{Ru}^{\mathrm{II} \star}={ }^{1} \mathrm{Co}^{\mathrm{III}}\right]$ and on the yield of $\left[{ }^{2} \mathrm{Ru}^{\mathrm{III}}={ }^{4} \mathrm{Co}{ }^{\mathrm{II}}(\mathrm{HS})\right]$ population via ET and SST. By the abovementioned relation between $\mathrm{X}$-ray emission intensity and spin state, the amplitude of $\Delta S_{\mathrm{XES}}(t)$ at $6.93 \mathrm{keV}, \gamma_{\mathrm{XES}}(t)$, monitors the formation kinetics of the charge-separated state where the reduced $\mathrm{Co}^{\mathrm{II}}$ species is in the $\mathrm{HS}$ state. Within the signal to noise of this experiment, the appearance of this species clearly occurs on the few picosecond timescale (red dots in Fig. 4b).

The electronic dynamics in the photoexcited dyad as followed by XES can now be contrasted to that observed with TOAS. While the TOAS signal has almost completely decayed at 2 ps, the XES difference signal is approaching its maximum amplitude only at $10 \mathrm{ps}$. In other words, the time taken by the electron to leave the bridge $(490 \pm 17 \mathrm{fs})$ cannot be identified with the time necessary for the $\left[{ }^{2} \mathrm{Ru}^{\mathrm{III}}={ }^{4} \mathrm{Co}^{\mathrm{II}}\right.$ (HS)] to appear (few picosecond). On leaving the bridge, the electron could localize on the distal portion of tpphz as a reduced ligand state or on the Co centre as a metal-centred state. Since no dynamics associated with the spectral fingerprints of bpy ${ }^{-}$or phen ${ }^{-}$(ref. 49) can be observed with TOAS in the ultraviolet and visible range, the intermediate species is optically dark. A sequential reaction

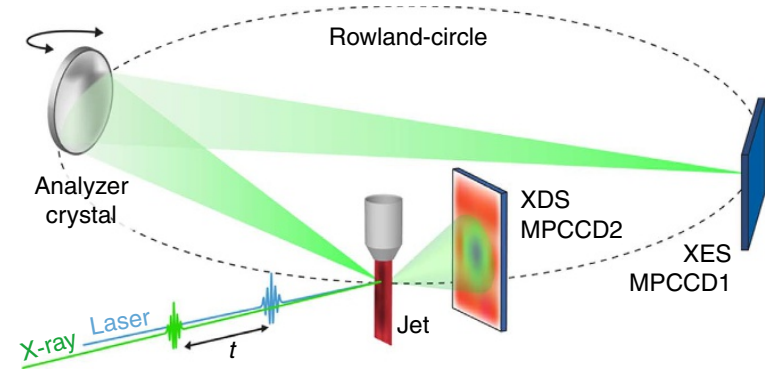

Figure 3 | Experimental setup. This optical pump-X-ray probe detection scheme combining XES and XDS on photoexcited species in solution was implemented at the SACLA XFEL facility. mechanism involving the ${ }^{2} \mathrm{Co}^{\mathrm{II}}$ (LS) electronically excited state can then be proposed, namely $\left[{ }^{1} \mathrm{Ru}^{\mathrm{II}}={ }^{1} \mathrm{Co} \mathrm{CII}^{\mathrm{II}}\right.$ (LS) $]+h v \rightarrow$ $\left[{ }^{2} \mathrm{Ru}^{\mathrm{III}}(=\bullet){ }^{1} \mathrm{Co}{ }^{\mathrm{III}}(\mathrm{LS})\right] \rightarrow\left[{ }^{2} \mathrm{Ru}{ }^{\mathrm{III}}={ }^{2} \mathrm{Co}^{\mathrm{II}}(\mathrm{LS})\right] \rightarrow\left[{ }^{2} \mathrm{Ru}^{\mathrm{III}}={ }^{4} \mathrm{Co}^{\mathrm{II}}\right.$ (HS)]. Within such a model, the formation rate of the intermediate species is locked to the $(490 \mathrm{fs})^{-1}$ decay rate of reduced pyrazine. The ratio of the XES amplitudes for $\left[{ }^{2} \mathrm{Ru}^{\mathrm{III}}={ }^{2} \mathrm{Co}{ }^{\mathrm{II}}(\mathrm{LS})\right] /\left[{ }^{2} \mathrm{Ru}^{\mathrm{III}}={ }^{4} \mathrm{Co}^{\mathrm{II}}(\mathrm{HS})\right]$ is fixed to $1 / 3$, which would be expected from the variations in FWHM for the XES signal of a ${ }^{2} \mathrm{Co}^{\mathrm{II}}$ (LS) $\rightarrow{ }^{4} \mathrm{Co}^{\mathrm{II}}$ (HS) SST. Fitting the kinetics trace $\gamma_{\mathrm{XES}}(t)$ (blue line in Fig. $4 \mathrm{~b}$, Table 1 in the Methods section and Supplementary Note 3) with the width of the Gaussian XFEL instrument response function (IRF) as a free parameter returns $1.9 \pm 0.6 \mathrm{ps}$ for the time constant of the step $\left[{ }^{2} \mathrm{Ru}{ }^{\mathrm{III}}={ }^{2}\right.$ $\left.\mathrm{Co}^{\mathrm{II}}(\mathrm{LS})\right] \rightarrow\left[{ }^{2} \mathrm{Ru}^{\mathrm{III}}={ }^{4} \mathrm{Co}^{\mathrm{II}}\right.$ (HS) $]$, and $520 \pm 410 \mathrm{fs}$ for the IRF, which is dominated by the temporal jitter between optical pump and X-ray probe. Since the measured ET rate is not instrument limited $^{50}$, the XES experiment is accessing the intrinsic timescale of the process. The temporal evolution of the $\left[{ }^{2} \mathrm{Ru}{ }^{I I I}(=\bullet)^{1} \mathrm{Co}^{\mathrm{III}}\right.$ (LS) $],\left[{ }^{2} \mathrm{Ru}^{\mathrm{III}}={ }^{2} \mathrm{Co}{ }^{\mathrm{II}}(\mathrm{LS})\right]$ and $\left[{ }^{2} \mathrm{Ru}^{\mathrm{III}}={ }^{4} \mathrm{Co}^{\mathrm{II}}(\mathrm{HS})\right]$ populations are shown in Fig. 4c (Supplementary Note 5). Summarizing the first conclusion reached in this study: the combination of femtosecond XES and TOAS measurements demonstrates that sub-picosecond ET occurs from the photoexcited Ru centre to an electronically excited state of the $\mathrm{Co}^{\mathrm{II}}$ centre, tentatively assigned as ${ }^{2} \mathrm{Co}{ }^{\mathrm{II}}(\mathrm{LS})$. This is followed by an $\sim 2$ ps electron localization, resulting in the formation of the $\left[{ }^{2} \mathrm{Ru}^{\mathrm{IIl}}={ }^{4} \mathrm{Co}{ }^{\mathrm{II}}\right.$ (HS) $]$ chargeseparated species. Since these timescales are typical of intramolecular vibration, energy redistribution and heat dissipation from vibrationally hot states to the environment, the complete characterization of the charge localization requires obtaining further information about the global structural changes and the interaction of the complex with its surroundings. Whereas this usually challenges optical spectroscopies, both aspects are readily amenable to investigation by the analysis of the XDS difference signal $\Delta S_{\mathrm{XDS}}(Q, t)$ acquired simultaneously with $\Delta S_{\mathrm{XES}}(t)$ in the present experiments.

Figure 5a shows the measured $\Delta S_{\mathrm{XDS}}(Q, t)$ after data reduction and background subtraction ${ }^{51}$ (Supplementary Notes 5 and 6; Supplementary Fig. 6). This signal is interpreted as arising primarily from the changes in solute structure $\Delta S_{\text {solute }}(Q, t)$ and from the bulk-solvent response $\Delta S_{\text {solvent }}(Q, t)$ (Supplementary Note 7$)$. The contribution $\Delta S_{\text {solute }}(Q, t)$ can be expressed as $\gamma_{\mathrm{XDS}}(t) \times \Delta S_{\mathrm{DFT}}(Q)$, where $\gamma_{\mathrm{XDS}}(t)$ is the time-dependent fraction of $\left[{ }^{2} \mathrm{Ru}^{\mathrm{III}}={ }^{4} \mathrm{Co}{ }^{\mathrm{II}}(\mathrm{HS})\right]$, and $\Delta S_{\mathrm{DFT}}(Q)$ is the profile calculated from the spin unrestricted DFT-optimized geometries of solvated $\left[{ }^{1} \mathrm{Ru}^{\mathrm{II}}={ }^{1} \mathrm{Co}{ }^{\mathrm{III}}\right.$ (LS) $]$ and $\left[{ }^{2} \mathrm{Ru}^{\mathrm{III}}={ }^{4} \mathrm{Co}^{\mathrm{II}}\right.$ (HS) $]$ (Supplementary Note 4). The distinctive negative $\operatorname{dip}$ at $Q=0.5 \AA^{-1}$ is associated to the Co-N bond length elongation by $\Delta R \sim 0.2 \AA$ in the ${ }^{4} \mathrm{Co}^{\mathrm{II}}$ HS state (Supplementary Fig. 7; Supplementary Fig. 10) ${ }^{34}$. For the time delays $t>3$ ps considered here, and for moderate temperature increase $\Delta T(t), \Delta S_{\text {solvent }}(Q, t)$ is a linear function of $\Delta T(t)$, by $\Delta S_{\text {solvent }}(Q, t)=\Delta T(t) \times\left.\frac{\partial S(Q)}{\partial T}\right|_{\rho}$, where $\left.\frac{\partial S(Q)}{\partial T}\right|_{\rho}$ is the difference scattering signal specific for $\mathrm{MeCN}$ at constant density $\rho$ (Supplementary Notes 9 and 10; Supplementary Fig. 9). This methodology for analysing $\Delta S_{\text {solvent }}(Q, t)$ has been developed at third-generation synchrotron facilities ${ }^{52,53}$ and has proven a robust and widely applicable approach ${ }^{32,54-56}$. In the present analysis, $\Delta S_{\mathrm{XDS}}(Q, t)$ was independently fitted for each $t$ to a linear combination of solute and solvent contributions broadened by the XFEL spectral function (Supplementary Note 8; Supplementary Fig. 8) with $\gamma_{\mathrm{XDS}}$ and $\Delta T$ as free parameters. Figure $5 \mathrm{~b}$ shows the experimental (black dots) and fitted (purple line) $\Delta S(Q)$ patterns for $t=25 \mathrm{ps}$, while Fig. $5 \mathrm{c}$ displays the contributions $\Delta S_{\mathrm{DFT}}(Q)$ (blue line) and $\left.\frac{\partial S(Q)}{\partial T}\right|_{\rho}$ (red line $)^{53}$ used in the fitting procedure (Supplementary Note 8). While a detailed structural refinement is 
a

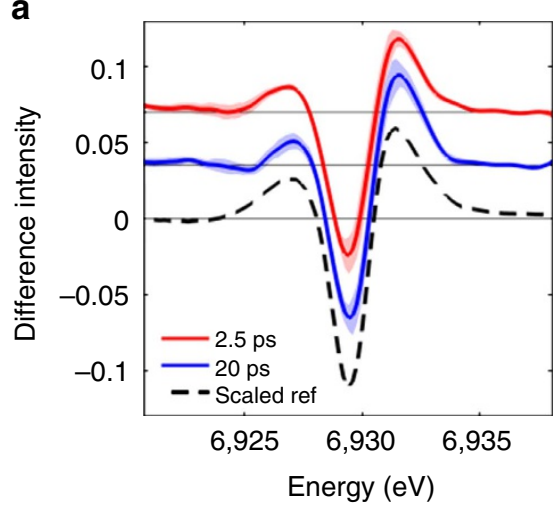

b

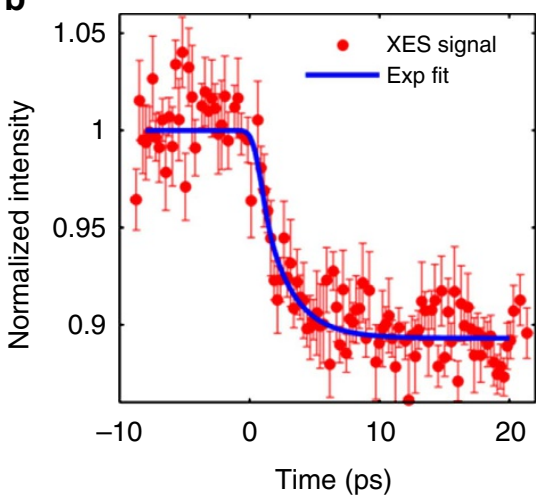

C

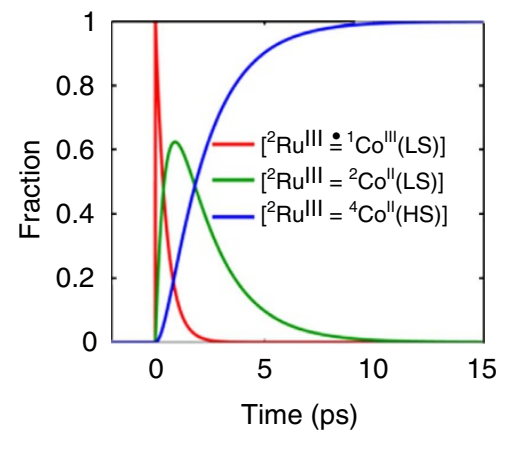

Figure 4 | Ultrafast X-ray emission spectroscopy. (a) Co $K \alpha_{1} \Delta S_{X E S}(t)$ at 2.5 (red) and 20 ps (blue) pump-probe delay. The shaded areas indicate the uncertainty level. The dashed black curve is the simulated reference for a ${ }^{1} \mathrm{Co}{ }^{\prime \prime l}(\mathrm{LS}) \rightarrow{ }^{4} \mathrm{Co}$ "l $(\mathrm{HS})$ conversion, scaled to the 20 ps trace. (b) Kinetic trace at $6.93 \mathrm{keV}$ (red dots) and single-exponential fit with a $1.9 \mathrm{ps}$ lifetime, broadened by a $520 \pm 410 \mathrm{fs}$ XFEL IRF (blue line). The error bars indicate the s.e. of

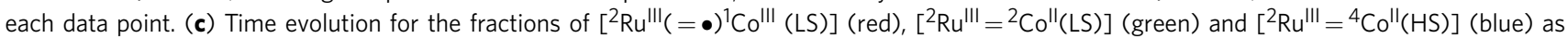
monitored by the combination of femtosecond TOAS and XES, where the initial fraction of $\left[{ }^{2} \mathrm{Ru}^{\text {IIII }}(=\bullet)^{1} \mathrm{Co} \mathrm{III}^{\text {III }}(\mathrm{LS})\right]$ was renormalized to 1 .
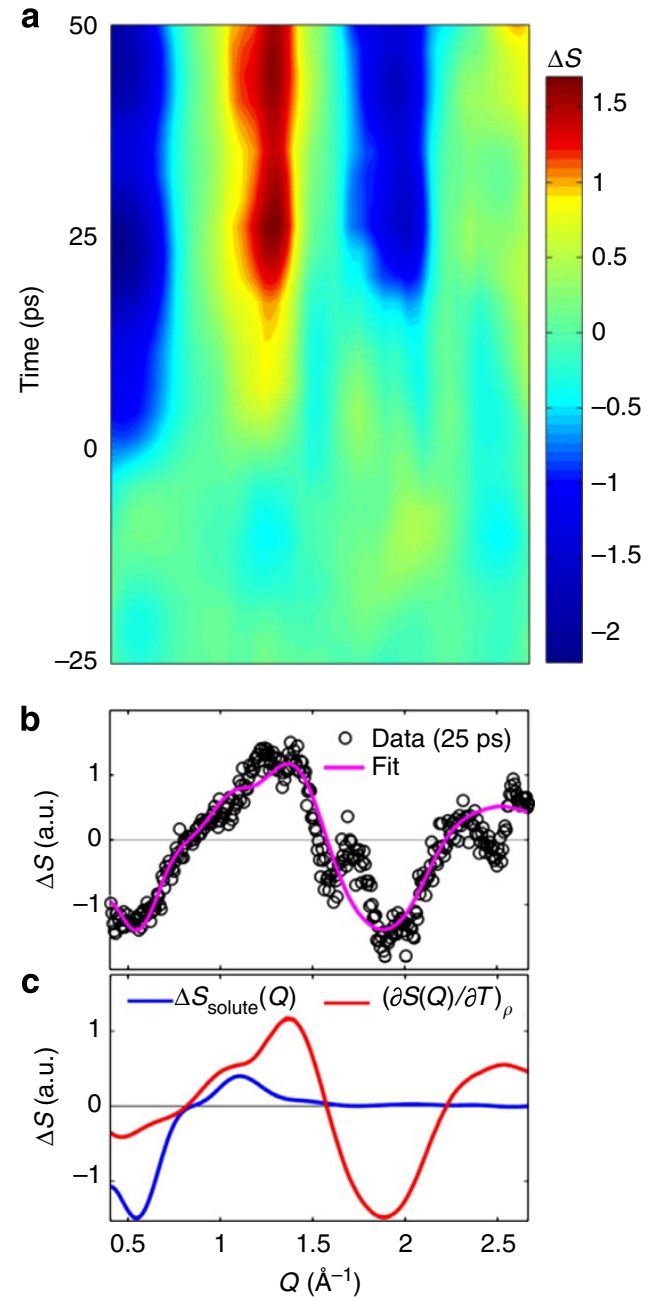

Figure 5 | Ultrafast X-ray diffuse scattering. (a) Median filtered $\Delta S_{X D S}(Q, t)$. (b) Experimental (black dots) and fitted (purple line) $\Delta S_{X D S}(Q, 25 \mathrm{ps})$. (c) Contributions from the solute (blue) and from the solvent (red).

beyond the signal-to-noise ratio obtainable at the time of the experiment, the two components shown in Fig. $5 \mathrm{c}$ represent the major contributions to the observed signal (Supplementary

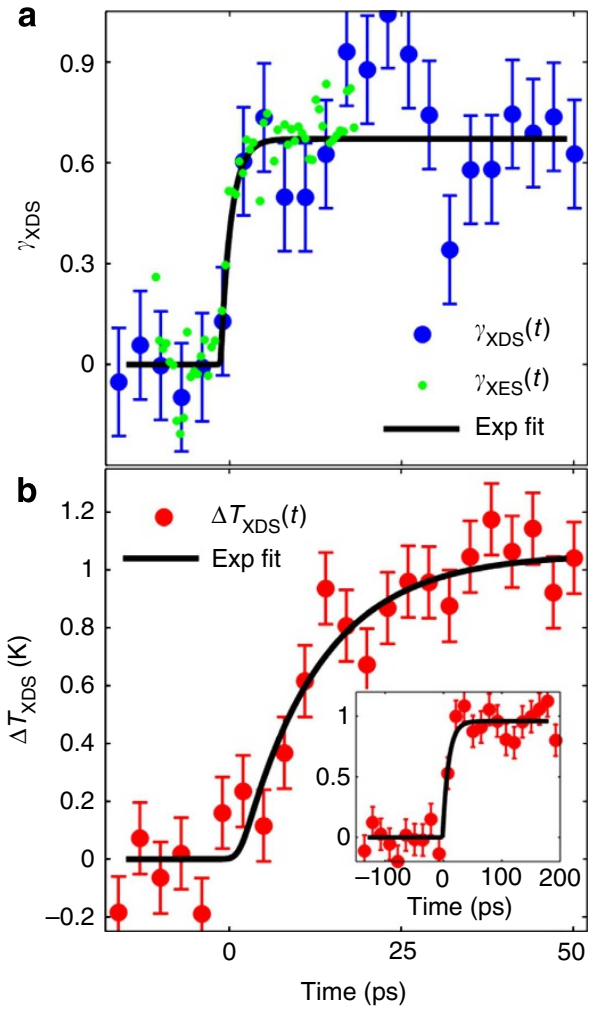

Figure 6 | Ultrafast XDS kinetics. (a) $\gamma_{\text {XDS }}(t)$ (blue dots) and $\gamma_{\text {XES }}(t)$ (green dots) as a function of pump-probe time delay. The singleexponential fits of $\gamma_{\mathrm{XDS}}(t)$ is indicated by the black line. (b) $\Delta T(t)$ kinetics (red dots), with its single-exponential fit (black line). The error bars six indicate the s.d. of the data points.

Note 11; Supplementary Fig. 10). Their amplitudes can thus be interpreted as the most significant kinetics of the structural rearrangements in the solute and the solvent. Figure $6 \mathrm{a}, \mathrm{b}$ contains the experimental $\gamma_{\mathrm{xDs}}(t)$ (blue dots) and $\Delta T(t)$ (red dots) and their respective fits after broadening by the $520 \pm 410$ fs XFEL IRF (black lines). The step-like increase of $\gamma_{\mathrm{XDS}}(t)$ to $67 \pm 4 \%$ for $t>0$ matches well the $65 \pm 8 \%$ obtained for $\gamma_{\mathrm{XES}}$ at $20 \mathrm{ps}$. The temporal evolution of $\Delta T(t)$ follows a single-exponential rise of $12 \pm 3 \mathrm{ps}$ and reaches a final value $\Delta T_{f}=1.0 \pm 0.1 \mathrm{~K}$. These XDS measurements therefore capture the structural changes of the 
solute and the early dynamics of impulsive solvent heating until thermalization. Comparing the timescales extracted independently from TOAS and simultaneous XES-XDS provide the second conclusion reached in this study: the initial charge transfer from the $\mathrm{Ru}$ centred to the electronically excited Co centre is 20 times faster than the local thermalization, and the charge localization, along with very large structural rearrangements that occur almost an order of magnitude faster than the thermalization of the hot molecule with its surroundings.

By combining ultrafast TOAS, XES and XDS measurements, all the timescales that characterize the non-equilibrated ET within the photoexcited $\left[{ }^{1} \mathrm{Ru}^{\mathrm{II}}={ }^{1} \mathrm{Co}{ }^{\mathrm{III}}\right.$ (LS) $]$ dyad are now resolved across several length scales, from the population of the optically excited Franck-Condon state to the fully equilibrated chargeseparated state. The TOAS and XES experiments track the subpicosecond electronic dynamics of the ET from the optically bright $\mathrm{Ru}$ donor to the optically dark Co acceptor. Three steps are identified on the few picosecond timescale: (1) quasi instantaneous ET from the photoexcited $\mathrm{Ru}$ centre to the tppz bridge ( $\tau<50 \mathrm{fs}$ ), (2) migration through the tpphz bridge ( $\tau \sim 0.5 \mathrm{ps}$ ) and (3) localization at the Co centre, which undergoes a SST ( $\tau \sim 1.9 \mathrm{ps})$. Contrasting the kinetics observed in the visible and the X-ray spectral range evidences that this last step involves an additional optically dark intermediate, tentatively assigned as the ${ }^{2} \mathrm{Co}{ }^{\mathrm{II}}(\mathrm{LS})$ state. The key role of electronically excited states in promoting such a process is further supported by DFT calculations. The electronic and geometric structures of $\left[{ }^{1} \mathrm{Ru}^{\mathrm{II}}=1\right.$ $\mathrm{Co}^{\mathrm{III}}$ (LS)] and $\left[{ }^{2} \mathrm{Ru}^{\mathrm{III}}={ }^{4} \mathrm{Co}^{\mathrm{II}}\right.$ (HS) $]$ solvated in $\mathrm{MeCN}$ were optimized using DFT. In the latter case of an open-shell system, the unrestricted formalism was applied to obtain the spin-up $(\alpha)$ and spin-down $(\beta)$ configurations (Supplementary Note 4 ). From the manifold of virtual unoccupied molecular orbitals (MOs) returned by the DFT calculations, it is clear that the electronic relaxation cascade from the Franck-Condon state should encompass the population of electronically excited species. The virtual MOs of $\left[{ }^{1} \mathrm{Ru}^{\mathrm{II}}={ }^{1} \mathrm{Co}{ }^{\mathrm{III}}\right.$ (LS) $]$ (Fig. 7a) mirror the frontier orbitals of the fully charge-separated $\left[{ }^{2} \mathrm{Ru}^{\mathrm{III}}={ }^{4} \mathrm{Co} \mathrm{II}^{\mathrm{II}}\right.$ (HS)] (Fig. 7b). Specifically, the (HOMO) $)_{\alpha}$ of $\left[{ }^{2} \mathrm{Ru}^{\mathrm{III}}={ }^{4} \mathrm{Co}^{\mathrm{II}}\right.$ (HS)] is very similar to the (LUMO +1$)$ of $\left[{ }^{1} \mathrm{Ru}^{\mathrm{II}}={ }^{1} \mathrm{Co}{ }^{\mathrm{III}}(\mathrm{LS})\right]$, while the $(\mathrm{LUMO})_{\alpha}$ and the $(\mathrm{LUMO}+1)_{\beta}$ of $\left[{ }^{2} \mathrm{Ru}{ }^{\mathrm{III}}={ }^{4} \mathrm{Co}^{\mathrm{II}}\right.$ (HS)] both resemble the bridge-centred (LUMO +2$)$ of $\left[{ }^{1} \mathrm{Ru}^{\mathrm{II}}={ }^{1} \mathrm{Co}{ }^{\mathrm{III}}\right.$ (LS) $]$. Confirming the assignment of the intermediate species as the
${ }^{2} \mathrm{Co}^{\mathrm{II}}(\mathrm{LS})$ state, and its role in promoting the ultrafast charge separation in this dyad calls for further investigations with the higher sensitivity and temporal resolution enabled by the ongoing development of timing tools ${ }^{57}$ and self-seeding schemes $^{58}$ at XFEL facilities.

On longer timescales ( $\sim 10 \mathrm{~s}$ of ps) and larger length scales ( $\sim 10 \mathrm{~s}$ of $\AA$ ), the XDS experiments track the structural changes around the Co centre $(\Delta R \sim 0.2 \AA)$ and the rate of solvent heating $(\tau \sim 12 \mathrm{ps})$. These simultaneous XES and XDS measurements constitute the first X-ray-based visualization of coupled photoinduced ET and structural rearrangements that both proceed much faster than the local equilibration. The time constant of solvent heating is in good agreement with the one obtained from TOAS ( $\tau \sim 8 \mathrm{ps}$ ) for the vibrational cooling of the optically bright states (that is, Ru based). The difference between these two rates can be partly ascribed to the existence of hot spots, where some degree of vibrational cooling takes place at the molecular level on the $100 \mathrm{fs}$ timescale, without significant energy transfer to the solvent. Collectively, the ultrafast TOAS, XES and XDS measurements provide direct experimental evidence that nonequilibrated ET mediated by electronically excited molecular states can yield charge-separated species that have undergone profound structural rearrangements through large-amplitude atomic motions, while still competing efficiently with intramolecular vibration energy redistribution and heat loss to the surroundings. The various steps and their respective timescales are illustrated in Fig. 8.

\section{Discussion}

The data quality achieved in this study indicates that these novel analytical X-ray tools are ready to be applied to the diagnostics of fully functional systems. The general implications of the results established in this work are now outlined. Dynamical information very similar to that obtained here is crucially lacking to rationalize the performances of most photoactive donor-acceptor assemblies and to improve the current bottom-up design strategies of photocatalysts. Maximizing the efficiency of photoconversion in supramolecular complexes requires optimizing three seemingly independent steps, namely panchromatic harvesting of sun light (step 1), rapid transfer of the excited electron to the acceptor site (step 2) and stabilization of the charge-separated species (step 3). a

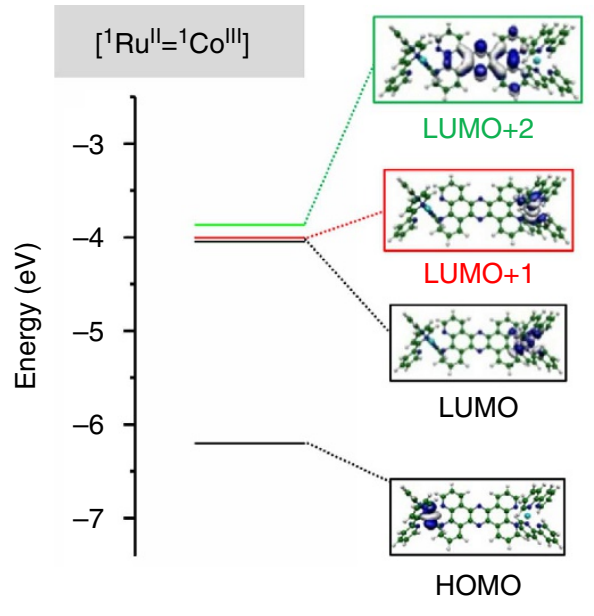

b
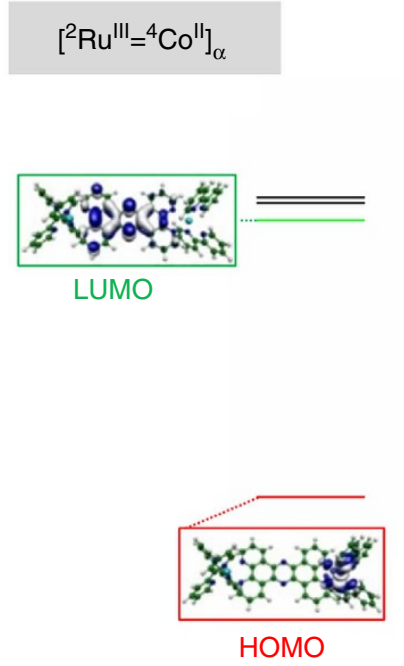

$\left[{ }^{2} \mathrm{Ru} u^{\prime \prime \prime}={ }^{4} \mathrm{Co}{ }^{\prime \prime}\right]_{\beta}$
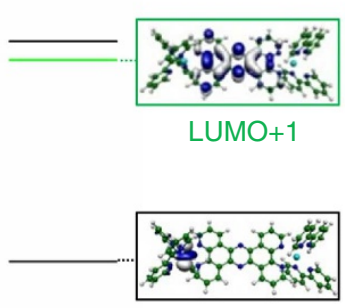

LUMO

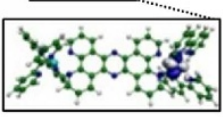

HOMO

Figure 7 | Low-lying electronically excited MOs and frontier MOs. These MOs have been obtained from DFT calculations for (a) the LS $\left[{ }^{1} \mathrm{Ru}{ }^{\prime \prime}={ }^{1} \mathrm{Co} \mathrm{O}^{\mathrm{III}}\right]$ and (b) the HS $\left[{ }^{2} \mathrm{Ru}{ }^{\prime \prime I}={ }^{4} \mathrm{Co}{ }^{\prime \prime}\right]_{\alpha}$ and $\left[{ }^{2} \mathrm{Ru}^{I I I}={ }^{4} \mathrm{Co}{ }^{\prime \prime}\right]_{\beta}$. 


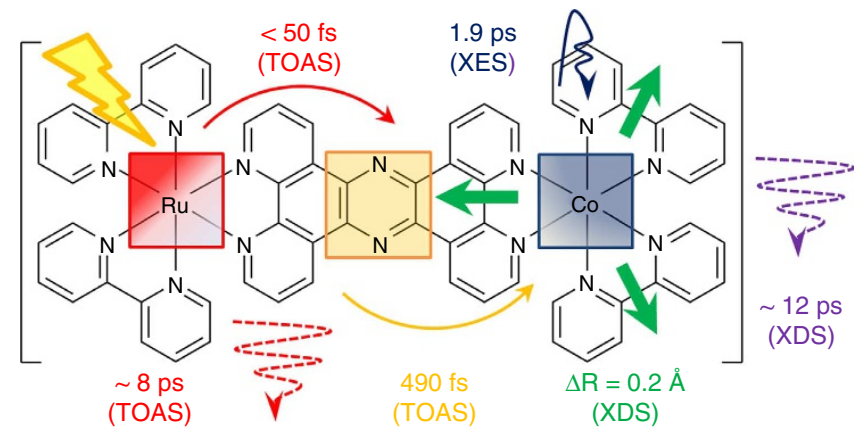

Figure 8 | Non-equilibrated ET across the photoexcited $\left[{ }^{1} \mathbf{R} \mathbf{u}^{\prime \prime}={ }^{\mathbf{1}} \mathbf{C o}^{\mathbf{I I I}}\right]$

dyad. The schematic summarizes the fundamental timescales, as obtained from TOAS and combined XES-XDS at the SACLA XFEL facility.

This sequence of steps results in a net metal-to-metal ET over large interatomic distances. Extending the absorbance without opening up detrimental loss channels, while promoting the ultrafast electronic localization onto the bridge, constitute intertwined targets in the elaboration of any photoactive dyad $^{59-61}$. From the feedback provided by TOAS about the ET rates, it is possible to tune the excited state manifolds of the donor-bridge units by introducing various electron donating/ withdrawing substituents on the peripheral/bridging ligands ${ }^{61}$ to realize concurrently step 1 and 2 for a given molecular architecture. A similar tailoring of the bridge-acceptor units has been hampered so far by the scarcity of techniques capable of probing the non-equilibrated ET dynamics at the catalytic sites, which are usually optically dark centres. This work establishes that measurements at XFEL facilities can now clock the ultrafast electronic localization at the acceptor site. It also unambiguously demonstrates that this ET rate cannot be generally identified with the quenching rate of the bridge-localized states monitored by TOAS, as commonly assumed. Therefore, tracking ET rates directly with element- and spin sensitivity should greatly contribute to rationalizing step 2 and the associated trends in structure-activity relationships. Advanced modelling with DFT, time-dependent DFT and QM/MM $\mathrm{MD}^{62}$ will have to be extensively employed to assist the mechanistic interpretations of the observations. Preliminary QM/MM (solute/solvent) equilibrium $\mathrm{MD}$ simulations of $\left[{ }^{1} \mathrm{Ru}^{\mathrm{II}}={ }^{1} \mathrm{Co} \mathrm{CII}^{\mathrm{III}}(\mathrm{LS})\right]$ and $\left[{ }^{2} \mathrm{Ru}^{\mathrm{III}}={ }^{4} \mathrm{Co}^{\mathrm{II}}\right.$ (HS) $]$ can already confirm the long-timesimulated structural change around the Co centre reported in this communication (Supplementary Note 12; Supplementary Fig. 11). Such theoretical guidelines will prove essential, since the predominant participation of highly excited states necessitates going beyond the frame of Marcus theory. For example, no clear-cut argumentation based on the driving force, reorganization energy or electronic coupling can yet be put forward to explain why the charge separation in $\left[{ }^{1} \mathrm{Ru}^{\mathrm{II}}={ }^{1} \mathrm{Co} \mathrm{CII}^{\mathrm{II}}\right.$ (LS)] is an order of magnitude faster than in closely related complexes (for example, $\left[{ }^{1} \mathrm{Ru}^{\mathrm{II}}={ }^{1} \mathrm{O} s^{\mathrm{III}}(\mathrm{LS})\right]^{35}$. The present experiments also reveal that the ET and the SST proceed much faster than the dissipation of excess energy to the environment. Moreover, the energy-storing ET step $\mathrm{Ru} \rightarrow \mathrm{Co}$ occurs on the same timescale as the intramolecular redistribution of vibrational energy, which is a prerequisite for full photon energy utilization. In other words, step 2 has the potential to produce 'hot' activated acceptors that can drive highly endoenergetic reactions. The systematic exploitation of this aspect will require extensive input from ultrafast X-ray experiments whenever the active site is optically dark. By merging the information obtained from ultrafast optical and X-ray experiments, it becomes possible to follow vectorial non-equilibrated ET throughout a dynamically
Table 1 | Analysis of the transient XES signal.

\begin{tabular}{lccccc} 
Model & $\boldsymbol{t}_{\mathbf{0}}$ (ps) & $\gamma_{\mathbf{0}}(\mathbf{p s})$ & $\tau_{\mathbf{1}}(\mathbf{p s})$ & $\boldsymbol{\sigma}(\mathbf{p s})$ & $\boldsymbol{P}$ \\
\hline $\mathrm{A}$ & $1.5 \pm 0.3$ & $0.66 \pm 0.02$ & - & $1.5 \pm 0.3$ & 0.04 \\
$\mathrm{~B}$ & $0.05 \pm 0.13$ & $0.67 \pm 0.02$ & $1.9 \pm 0.5$ & $0.52 \pm 0.41$ & 0.50
\end{tabular}

The fitting parameters were $t_{0}$ (time 0 ), $\gamma_{0}$ (initial excitation fraction), $\tau_{1}$ (free rate constant) and $\sigma$ (width of the Gaussian broadening) for models A and B. The corresponding $p$ parameters are given in the last column.

evolving molecular architecture, as it interacts with the immediate surroundings. Optimizing the sequence of step 1,2 and 3 can now be approached as a single-integrated task ${ }^{63,64}$.

In conclusion, the two emerging femtosecond $\mathrm{X}$-ray measurement techniques XES and XDS have been combined at the SACLA XFEL facility to unveil the fundamental timescales of non-equilibrated ET in a bimetallic donor-acceptor complex. XES follows the ultrafast reduction of the Co centre and the accompanying spin-state transition that takes only about $2 \mathrm{ps}$. Correlation with optical measurements demonstrates the participation of at least one optically dark step. XDS catches the structural changes of the solute, as well as the onset of the structural response arising from ultrafast solute-mediated solvent heating. The stabilization of the charge-separated state over $13 \AA$ is determined to be faster than the large structural reorganization of the complex and dissipation of excess energy in the surroundings. Insights provided by DFT calculations suggest that the ultrafast ET is promoted by electronically excited molecular states, a key mechanistic feature of this non-adiabatic process. Ascertaining the mechanisms of non-equilibrated ET in the homogeneous phase is paving the way to controlling and manipulating hot ET, which is generally foreseen as one of the routes to follow if we are to match the unrivalled efficiency of natural photosynthesis.

\section{Methods}

Materials. The dyad studied in this work is the dinuclear complex [(bpy $)_{2} \mathrm{Ru}^{\mathrm{II}}$ $\left.(\text { tpphz })^{1} \mathrm{Co}^{\mathrm{III}}(\mathrm{bpy})_{2}\right]^{5+}$ (abbreviated as $\left[\mathrm{Ru}^{\mathrm{II}}={ }^{1} \mathrm{Co}^{\mathrm{III}}\right]$ ) where 'bpy' is $2,2^{\prime}$ -

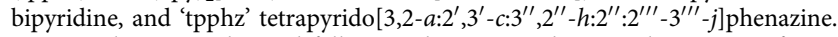
Its $\mathrm{PF}_{6}$ salt was synthesized following the improved protocol given in ref. 34 .

Femtosecond transient absorption spectroscopy. The femtosecond laser setup used for transient absorption spectroscopy has been previously described ${ }^{65}$. The excitation beam was depolarized and set to $400 \mathrm{~nm}$. The fitting procedure is described in Supplementary Note 1 with Supplementary Fig. 1.

Experimental X-ray setup. An optical pump-X-ray probe setup combining XES and XDS was implemented at beamline BL 3 of the SACLA XFEL facility, Japan (Fig. 1a). A $6 \mathrm{mM}$ solution of $\left[{ }^{1} \mathrm{Ru}^{\mathrm{II}}={ }^{1} \mathrm{Co}^{\mathrm{III}}\right]$ in acetonitrile $(\mathrm{MeCN})$ was continuously circulated in a free-flowing planar liquid sheet $(100 \mu \mathrm{m})$. The molecules were optically excited at $400 \mathrm{~nm}$ (60 fs pulse length, $500 \mu \mathrm{m}$ FWHM focus spot and $220 \mu \mathrm{J}$ per pulse). The probe consisted of $8 \mathrm{keV}$ X-ray pulses ( $10 \mathrm{fs}$ pulse length, $0.3 \%$ bw, $450 \mu \mathrm{m}$ FWHM beam size and $10^{10}$ photons per pulse) generated by SACLA. Both laser and X-rays were operated at a $10 \mathrm{~Hz}$ repetition frequency. The two beams crossed under a $10^{\circ}$ angle in the horizontal plane. The effective time resolution was given by the shot-to-shot jitter between the optical and XFEL pulses.

A $4^{\prime \prime}$ diameter spherically bent $\mathrm{Si}(531)$ analyser crystal (1,010 $\mathrm{mm}$ bent radius) working in the Rowland circle geometry was used to measure the $\mathrm{Co} \mathrm{K} \alpha_{1}$ emission maximum at $6.93 \mathrm{keV}$. It was placed at a scattering angle of $130^{\circ}$, to resolve and focus the X-ray emission at a Bragg angle of $77^{\circ}$ in the horizontal plane. This signal was detected by a multiport-charged-coupled device (MPCCD) area detector. Rotation of the analyser crystal with concurrent movement of the MPCCD allowed for energy (wavelength) selection. The XDS signal was recorded on a second MPCCD detector placed $3.5 \mathrm{~cm}$ behind the sample (with the direct beam blocked), allowing for detection in a Q-range spanning from 0.4 to $3.2 \AA^{-1}$. The XES and XDS detectors were read out after every X-ray pulse. The signal of interest was extracted from a differential measurement with and without laser irradiation on the sample, that is, as a [laser ${ }_{\mathrm{ON}}(t)-$ laser $_{\mathrm{OFF}}$ ] traces. Two types of scans (kinetic scans and energy scanes) were acquired. For the kinetic scans, the time delay $t$ between the laser and the X-ray pulse was varied by steps of 0.3 (for XES measurements), 
3 (for combined XES/XDS measurements) or 10 (for combined XES/XDS measurements). The signal was integrated for $20 \mathrm{~s}$ (corresponding to $200 \mathrm{X}$-ray pulses, with the detector signal read out for each individual pulse). For the scans with a 0.3 ps step size, negative delays of -100 ps (corresponding to the X-rays arriving 100 ps before the laser pump) were interspaced such that every fourth delay was a laser ${ }_{\mathrm{OFF}}$ measurement allowing for correction of any long-term drifts in the signal during the acquisition.

For the energy scans of the XES intensity at constant $t$, the signal was integrated for $4 \mathrm{~s}$ (corresponding to $40 \mathrm{X}$-ray pulses) at each energy point.

XES data analysis. The fine structure of the $\mathrm{K}_{\alpha}$ spectra originates from multiplet and spin orbit interactions. In transition metal systems, they are highly sensitive to the oxidation state and to the number of unpaired electrons $s^{24,26,66}$. The twodimensional images containing the XES signal were recorded by the MPCCD detector at the focus of the analyser crystal. The images were corrected, and the photons of each exposure were explicitly counted for every X-ray pulse as described in Supplementary Note 2 . The $\mathrm{K} \alpha_{1}$ spectra were constructed from the energy scans, resulting in the transient XES signal $\Delta S_{\mathrm{XES}}(t)$ curves shown in Fig. 2a (main text). A lineshape analysis is typically applied to extract the charge and spin information from such XES data ${ }^{26,67-69}$. However, the short effective data collection time of the experiments reported in this work did not permit to obtain a sufficiently large set of spectra with known references and good statistics to fully exploit this approach. It was nevertheless indirectly applied to follow the spectral variations, since upon increase in spin state ${ }^{26}$, the $K \alpha_{1}$ spectra of Co undergoes a clear broadening. As such, the linewidth in $\mathrm{K} \alpha_{1}$ spectra can also be used to calibrate the charge and spin momentum $S$ on the Co centre. For the data presented in Fig. $4 \mathrm{a}$, the difference in linewidth between the ground state and the photoexcited state is $0.6 \mathrm{eV}$. This value corresponds to a spin-state change $\Delta S$ of 1.5 . Since $S=0$ in the initial ${ }^{1} \mathrm{Co}^{\mathrm{III}}$ state, the final spin state is $S=3 / 2$, that is, that of a $\mathrm{HS}^{4} \mathrm{Co}^{\mathrm{II}}$ (Supplementary Fig. 4). Since the total $\mathrm{K} \alpha_{1}$ emission intensity does not depend on the charge and spin state, the relative changes in the width of the emission line for the different Co species directly result in an inverse lowering of the maximum emission intensity, that is, the peak height. This is the parameter measured and plotted in the kinetic traces presented in Fig. $4 \mathrm{~b}$ and Supplementary Fig. 5.

Kinetic models for the XES data. Two kinetic models of charge and spin dynamics have been tested against the observed XES kinetics. In model A, concerted ET and SST ${ }^{1} \mathrm{Co}{ }^{\mathrm{III}}(\mathrm{LS}) \rightarrow{ }^{4} \mathrm{Co}{ }^{\mathrm{II}}(\mathrm{HS})$ take place with an overall rate constant $1 / \tau_{0}$, where $\tau_{0}=(490 \mathrm{fs})^{-1}$ is the lifetime of the reduced bridge as measured with TOAS. Starting with an initial excitation fraction of $\gamma_{0}$, the time-dependent concentrations of the transient species for this model $\mathrm{A}$ is:

$$
\left[\mathrm{Co}^{\mathrm{II}}(\mathrm{HS})\right]=\gamma_{0}\left(1-e^{-t / \tau_{0}}\right)
$$

In model $\mathrm{B}$, sequential ET ${ }^{1} \mathrm{Co}^{\mathrm{III}}(\mathrm{LS}) \rightarrow{ }^{2} \mathrm{Co}^{\mathrm{II}}(\mathrm{LS})$ with a rate constant $1 / \tau_{0}$ matching the decay rate of reduced bridge from TOAS is followed by the $\mathrm{SST}^{2} \mathrm{Co}^{\mathrm{II}}(\mathrm{LS}) \rightarrow{ }^{4} \mathrm{Co}^{\mathrm{II}}(\mathrm{HS})$ with a free rate constant $1 / \tau_{1}$, resulting in ${ }^{1} \mathrm{Co}^{\mathrm{III}}$ $(\mathrm{LS}) \rightarrow{ }^{2} \mathrm{Co}{ }^{\mathrm{II}}$ (LS) $\rightarrow{ }^{4} \mathrm{Co}^{\mathrm{II}}$ (HS)Starting with an initial excitation fraction of $\gamma_{0}$, the time-dependent concentrations of the transient species for this model are:

$$
\begin{gathered}
{\left[\mathrm{Co}^{\mathrm{II}}(\mathrm{LS})\right]=\gamma_{0} \frac{\tau_{1}}{\tau_{0}-\tau_{1}}\left(e^{-t / \tau_{0}}-e^{-t / \tau_{1}}\right)} \\
{\left[\mathrm{Co}^{\mathrm{II}}(\mathrm{HS})\right]=\gamma_{0}\left(1-\frac{\left(e^{-t / \tau_{0}} / \tau_{1}\right)-\left(e^{-t / \tau_{1}} / \tau_{0}\right)}{1 / \tau_{1}-1 / \tau_{0}}\right)}
\end{gathered}
$$

The thermally activated back ET to the ground state was omitted since the lifetime of the charge-separated $\left[{ }^{2} \mathrm{Ru}^{\mathrm{III}}={ }^{4} \mathrm{Co}^{\mathrm{II}}(\mathrm{HS})\right]$ is $45 \mathrm{~ns}^{35}$, that is, three orders of magnitude longer than the temporal window studied in this work. The time evolution of the XES signals was then modelled by convoluting the transient concentrations introduced above with a Gaussian IRF. Taking model A as an example, the resulting kinetics were given by:

$$
\begin{aligned}
I(\Delta t) & =I\left(t-t_{0}\right) \\
& =\gamma_{0} \int_{-\infty}^{\infty} \frac{1}{\sigma \sqrt{2 \pi}} e^{-y^{2} / 2 \sigma^{2}} H\left(t-t_{0}-y\right)\left(1-e^{-\left(t-t_{0}-y\right) / \tau_{0}}\right) d y
\end{aligned}
$$

where $\sigma$ is the width of the Gaussian broadening, $t_{0}$ is time $0, \gamma_{0}$ is the amplitude of the difference signal at $t_{0}$ and $H$ is the Heaviside step function.

The corrected Akaike information criterion (AICc) is then used to compare the relative goodness of the fit weighted by the model complexity ${ }^{70}$. Briefly, the AIC is introduced as:

$$
\mathrm{AIC}=2 k-2 \ln (L),
$$

where $k$ is the number of fit parameters and $L$ is the maximized likelihood value of the fit defined as:

$$
\ln (L)=c-\frac{1}{2} \sum_{i=1}^{N} \frac{\left(y_{i}-f\left(x_{i}\right)\right)^{2}}{\sigma_{i}^{2}}=c-\frac{\chi^{2}}{2},
$$

where $N$ is the number of data points, $y_{i}$ is the measured value, $f\left(x_{i}\right)$ is the fit value, $\sigma_{i}$ is the standard deviation of the measured values and $c$ is a constant. It has been shown that, for small data sets, the bias-corrected AIC value (AICc) should be used for model comparison:

$$
\mathrm{AICc}=\mathrm{AIC}+\frac{2 k(k+1)}{n-k-1}
$$

The direct relation of the AIC value to the maximized likelihood entails that it can be directly determined from the reduced $\chi^{2}$ :

$$
\mathrm{AIC}=\chi^{2}+2 k
$$

Considering two fitting models ( 1 and 2) of $\mathrm{AICc}$ values $\mathrm{AICc}_{1}$ and $\mathrm{AICc}_{2}$, the probability $p$ that model 1 is the most likely of the two models is given by:

$$
p=\frac{e^{-\Delta_{\mathrm{AICl}} / 2}}{1+e^{-\Delta_{\mathrm{AICl}} / 2}}
$$

where $\Delta_{\mathrm{AICc}}=\mathrm{AICc}_{1}-\mathrm{AICc}_{2}$.

The best fit parameters for the two models introduced above are listed in Table 1, along with their AICc-derived probabilities $p_{\mathrm{A}}$ and $p_{\mathrm{B}}$, taking $\mathrm{B}$ as reference. Considering these probabilities, there is substantial evidence for rejecting A in favour of B. The Supplementary Fig. 5 shows the fits resulting from models A (cyan) and B (blue) against the XES data. Model A systematically underestimates the data in both the region around $t_{0}$, as well as the region between 2 and $6 \mathrm{ps}$.

XDS data analysis. Two-dimensional X-ray scattering images were recorded by the MPCCD detector behind the sample. These images were corrected (for sample absorption, solid angle coverage and detector efficiency as a function of angle and polarization), azimuthally integrated and individually scaled to yield onedimensional radial curves $S(Q, t)$, which were used to construct the difference X-ray scattering signals $\Delta S_{\mathrm{XDS}}(Q, t)=\left[S_{\mathrm{ON}}(Q, t)-S_{\mathrm{OFF}}(Q)\right]$ for each time delay $t$, as described in detail in Supplementary Note 5 . The set of $\Delta S_{\mathrm{XDS}}(Q, t)$ was subsequently analysed in a framework developed for treating such data containing a significant contribution from a fluctuating background. The method relies on fitting SVD-detected background components simultaneously with components calculated form a physical model (see main text) to $\Delta S_{\mathrm{XDS}}(Q, t)$ for each $t$. This approach, described in detail in ref. 51, is summarized as applied to the present data sets in Supplementary Notes 6 and 8.

\section{References}

1. Rosspeintner, A., Lang, B. \& Vauthey, E. Ultrafast photochemistry in liquids. Annu. Rev. Phys. Chem. 64, 247-271 (2013).

2. Heitele, H. Dynamic solvent effects on electron-transfer reactions. Angew. Chem. Int. Ed. 32, 359-377 (1993).

3. Barbara, P. F., Walker, G. C. \& Smith, T. P. Vibrational modes and the dynamic solvent effect in electron and proton transfer. Science 256, 975-981 (1992).

4. Rosker, M. J., Dantus, M. \& Zewail, A. H. Femtosecond clocking of the chemical bond. Science 241, 1200-1202 (1988).

5. Jimenez, R., Fleming, G. R., Kumar, P. V. \& Maroncelli, M. Femtosecond solvation dynamics of water. Nature 369, 471-473 (1994).

6. Hamm, P. et al. Femtosecond infrared spectroscopy of reaction centers from Rhodobacter sphaeroides between 1000 and $1800 \mathrm{~cm}-1$. Proc. Nat Acad. Sci. USA. 92, 1826-1830 (1995).

7. Walla, P. J. et al. Femtosecond dynamics of the forbidden carotenoid S1 state in light-harvesting complexes of purple bacteria observed after two-photon excitation. Proc. Natl Acad. Sci. USA 97, 10808-10813 (2000).

8. Schoenlein, R. W., Peteanu, L. A., Mathies, R. A. \& Shank, C. V. The first step in vision: Femtosecond isomerization of rhodopsin. Science 254, 412-415 (1991).

9. Mokhtari, A., Cong, P., Herek, J. L. \& Zewail, A. H. Direct femtosecond mapping of trajectories in a chemical reaction. Nature 348, 225-227 (1990).

10. Damrauer, N. H. et al. Femtosecond dynamics of excited-state evolution in $[\mathrm{Ru}(\mathrm{bpy}) 3] 2+$. Science 275, 54-57 (1997).

11. Wasielewski, M. R. Photoinduced electron transfer in supramolecular systems for artificial photosynthesis. Chem. Rev. 92, 435-461 (1992).

12. Cannizzo, A. et al. Broadband femtosecond fluorescence spectroscopy of [Ru(bpy) 3]2 +. Angew. Chem. Int. Ed. 45, 3174-3176 (2006).

13. Bazant, M. Z. Theory of chemical kinetics and charge transfer based on nonequilibrium thermodynamics. Acc. Chem. Res. 46, 1144-1160 (2013).

14. Tidsale, W. A. et al. Hot-electron transfer from semiconductor nanocrystals. Science 328, 1543-1547 (2010).

15. Chang, J., Fedro, A. J. \& Van Veenendaal, M. Ultrafast X-ray spectroscopy as a probe of nonequilibrium dynamics in ruthenium complexes. Chem. Phys. 407, 65-70 (2012).

16. Elsaesser, T. \& Kaiser, W. Vibrational and vibronic relaxation of large polyatomic molecules in liquids. Annu. Rev. Phys. Chem. 42, 83-107 (1991).

17. Erk, B. et al. Imaging charge transfer in iodomethane upon X-ray photoabsorption. Science 345, 288-291 (2014).

18. Lemke, H. T. et al. Femtosecond X-ray absorption spectroscopy at a hard X-ray free electron laser: application to spin crossover dynamics. J. Phys. Chem. A 117, 735-740 (2012). 
19. Zhang, W. et al. Tracking excited-state charge and spin dynamics in iron coordination complexes. Nature 509, 345-348 (2014).

20. Kern, T. et al. Taking snapshots of photosynthetic water oxidation using femtosecond X-ray diffraction and spectroscopy. Nat. Commun. 5, 4371 (2014).

21. Dell'Angela, M. et al. Real-time observation of surface bond breaking with an X-ray laser. Science 339, 1302-1305 (2013).

22. Siefermann, K. R. et al. Atomic-scale perspective of ultrafast charge transfer at a dye-semiconductor interface. J. Phys. Chem. Lett. 5, 2753-2759 (2014).

23. Ishikawa, T. et al. A compact X-ray free-electron laser emitting in the sub-ångström region. Nat. Photon. 6, 540-544 (2012).

24. Glatzel, P. \& Bergmann, U. High resolution 1s core hole X-ray spectroscopy in $3 \mathrm{~d}$ transition metal complexes - Electronic and structural information. Coord. Chem. Rev. 249, 65-95 (2005)

25. Rovezzi, G. \& Glatzel, P. Hard X-ray emission spectroscopy: a powerful tool for the characterization of magnetic semiconductors. Semicond. Sci. Technol. 29, 023002 (2014)

26. Vankó, G. et al. Probing the 3D spin momentum with X-ray emission spectroscopy: the case of molecular-spin transition. J. Phys. Chem. B 110, 11647-11653 (2006)

27. Henriksen, N. E. \& Møller, K. B. On the theory of time-resolved X-ray diffraction. J. Phys. Chem. B 112, 558-567 (2008).

28. Lorenz, U., Møller, K. B. \& Henriksen, N. E. On the interpretation of timeresolved anisotropic diffraction patterns. New J. Phys. 12, 113022 (2010).

29. Lorenz, U., Møller, K. B. \& Henriksen, N. E. Theory of time-resolved inelastic X-ray diffraction. Phys. Rev. A 81, 023422 (2010).

30. Møller, K. B. \& Henriksen, N. E. Time-resolved X-ray diffraction: the dynamics of the chemical bond. Struct. Bond. 142, 185-211 (2012).

31. Ihee, H. et al. Ultrafast X-ray scattering: structural dynamics from diatomic to protein molecules. Int. Rev. Phys. Chem. 12, 453-520 (2010).

32. Haldrup, K., Christensen, M. \& Meedom Nielsen, M. Analysis of time-resolved $\mathrm{X}$-ray scattering data from solution-state systems. Acta Crystallogr. A 66, 261-269 (2010)

33. Torieda, H., Nozaki, K., Yoshimura, A. \& Ohno, T. Low quantum yields of relaxed electron transfer products of moderately coupled ruthenium(II)cobalt(III) compounds on the subpicosecond laser excitation. J. Phys. Chem. A 108, 4819-4829 (2004).

34. Canton, S. E. et al. Toward highlighting the ultrafast electron transfer dynamics at the optically dark sites of photocatalysts. J. Phys. Chem. Lett. 4, 1972-1976 (2013).

35. Chiorboli, C., Rodgers, M. A. J. \& Scandola, F. Ultrafast processes in bimetallic dyads with extended aromatic bridges. Energy and electron transfer pathways in tetrapyridophenazine-bridged complexes. J. Am. Chem. Soc. 125, 483-491 (2013).

36. Bolger, J., Gourdon, A., Ishow, H. \& Launay, J.-P. Mononuclear and binuclear tetrapyrido[3,2-a: $\left.2^{\prime}, 3^{\prime}-\mathrm{c}: 3^{\prime \prime}, 2^{\prime \prime}-\mathrm{A}: 2^{\prime \prime \prime}, 3^{\prime \prime \prime}-\mathrm{j}\right]$ phenazine (tpphz) ruthenium and osmium complexes. Inorg. Chem. 35, 2937-2944 (1996).

37. Flamigni, L. et al. Excited-state interconversion between emissive MLCT levels in a dinuclear $\mathrm{Ru}(\mathrm{II})$ complex containing a bridging ligand with an extended $\pi$ system. Chem. Commun. 1185-1186 (2000).

38. Luo, S. P. et al. Photocatalytic water reduction with copper-based photosensitizers: a noble-metal-free system. Angew. Chem. Int. Ed. 52, 419-423 (2012).

39. Duan, L. et al. A molecular ruthenium catalyst with water-oxidation activity comparable to that of photosystem II. Nat. Chem. 4, 418-423 (2012).

40. Mulfort, K. L., Mukherjee, A., Kokhan, O., Du, P. \& Tiede, D. M. Structurefunction analyses of solar fuels catalysts using in situ X-ray scattering. Coord. Chem. Rev. 42, 2215-2227 (2013).

41. Utschig, L. M., Silver, S. C., Mulfort, K. L. \& Tiede, D. M. Nature-driven photochemistry for catalytic solar hydrogen production: a photosystem I-transition metal catalyst hybrid. J. Am. Chem. Soc. 133, 16334-16337 (2011).

42. Benkö, G., Kallioinen, J., Korppi-Tommola, J. E. I., Yartsev, A. P. \& Sundström, V. Photoinduced ultrafast dye-to-semiconductor electron injection from nonthermalized and thermalized donor states. J. Am. Chem. Soc. 124, 489-493 (2002).

43. Liu, Y. et al. Towards longer-lived metal-to-ligand charge transfer states of iron(II) complexes: an N-heterocyclic carbene approach. Chem. Commun. 49, 6412-6414 (2013).

44. Kern, T. et al. Simultaneous femtosecond X-ray spectroscopy and diffraction of photosystem II at room temperature. Science 340, 491-495 (2013).

45. Haldrup, K. et al. Guest-host interactions investigated by time-resolved X-ray spectroscopies and scattering at $\mathrm{MHz}$ rates: solvation dynamics and photoinduced spin transition in aqueous Fe(bipy) $32+$. J. Phys. Chem. A 116, 9878-9887 (2012).

46. Vankó, G., Renz, F., Molnár, G., Neisius, T. \& Kárpáti, S. Hard-X-ray-induced excited-spin-state trapping. Angew. Chem. Int. Ed. 46, 5306-5309 (2007).

47. Vankó, G. et al. Picosecond time-resolved X-ray emission spectroscopy: ultrafast spin-state determination in an iron complex. Angew. Chem. Int. Ed. 49, 5910-5912 (2010)
48. Vankó, G. et al. Spin-state studies with XES and RIXS: From static to ultrafast. J. Electron Spectrosc. Relat. Phen. 188, 166-171 (2013).

49. Záliš, S. et al. Origin of electronic absorption spectra of MLCT-excited and one-electron reduced 2,2'-bipyridine and 1,10-phenanthroline complexes. Inorg. Chim. Acta 374, 578-585 (2011).

50. Inubushi, Y. et al. Determination of the pulse duration of an X-Ray free electron laser using highly resolved single-shot spectra. Phys. Rev. Lett. 109, 144801 (2012).

51. Haldrup, K. Singular value decomposition as a tool for background corrections in time-resolved XFEL scattering data. Philos. Trans. R Soc. Lond. B Biol. Sci. 369, 20130336 (2014).

52. Cammarata, M. et al. Impulsive solvent heating probed by picosecond X-ray diffraction. J. Chem. Phys. 124, 124504 (2006).

53. Kjær, K. S. et al. Introducing a standard method for experimental determination of the solvent response in laser pump, X-ray probe time-resolved wide-angle X-ray scattering experiments on systems in solution. Phys. Chem. Chem. Phys. 15, 15003-15016 (2013).

54. Kong, Q. et al. Theoretical study of the triplet excited state of PtPOP and the exciplexes M-PtPOP $(\mathrm{M}=\mathrm{Tl}, \mathrm{Ag})$ in solution and comparison with ultrafast X-ray scattering results. Chem. Phys. 393, 117-122 (2012).

55. Lee, J. H. et al. Filming the birth of molecules and accompanying solvent rearrangement. J. Am. Chem. Soc. 135, 3255-3261 (2013).

56. Borfecchia, E. et al. Monitoring excited state dynamics in cis$[\mathrm{Ru}(\mathrm{bpy}) 2(\mathrm{py}) 2] 2+$ by ultrafast synchrotron techniques. Catal. Today 229, 34-45 (2014).

57. Harmand, M. et al. Achieving few-femtosecond time-sorting at hard X-ray freeelectron lasers. Nat. Photon. 7, 215-218 (2013).

58. Amann, J. et al. Demonstration of self-seeding in a hard-X-ray free-electron laser. Nat. Photon. 6, 693-698 (2012).

59. Tschierlei, S. et al. Photophysics of an intramolecular hydrogen-evolving Ru-Pd photocatalyst. Chemistry 15, 7678-7688 (2009).

60. Tschierlei, S. et al. Photochemical fate: the first step determines efficiency of $\mathrm{H} 2$ formation with a supramolecular. Angew. Chem. Int. Ed. 49, 3981-3984 (2010).

61. Schulz, M., Karnahl, M., Schwalbe, M. \& Vos, J. G. The role of the bridging ligand in photocatalytic supramolecular assemblies for the reduction of protons and carbon dioxide Coord. Chem. Rev. 256, 1682-1705 (2012).

62. Dohn, A. O. et al. Direct dynamics studies of a binuclear metal complex in solution: the interplay between vibrational relaxation, coherence, and solvent effects. J. Phys. Chem. Lett. 5, 2414-2418 (2014).

63. Mulfort, K. L. \& Tiede, D. M. Supramolecular cobaloxime assemblies for h2 photocatalysis: An initial solution state structure-function analysis. J. Phys. Chem. B 114, 14572-14581 (2010).

64. Lei, P. et al. The role of colloid formation in the photoinduced $\mathrm{H} 2$ production with a RuII-PdII supramolecular complex: a study by GC, XPS, and TEM. J. Am. Chem. Soc. 130, 26-27 (2010)

65. Burdett, J. J., Gosztola, D. \& Bardeen, C. J. The dependence of singlet exciton relaxation on excitation density and temperature in polycrystalline tetracene thin films: kinetic evidence for a dark intermediate state and implications for singlet fission. J. Chem. Phys. 135, 214508 (2011).

66. Peng, G. et al. High-resolution manganese X-ray fluorescence spectroscopy. Oxidation state and spin-state sensitivity. J. Am. Chem. Soc. 116, 2914-2920 (1994).

67. Rueff, J. P. et al. Magnetism of Invar alloys under pressure examined by inelastic X-ray scattering. Phys. Rev. B 63, 132409 (2001).

68. Sikora, M., Knizek, K., Kapusta, C. \& Glatzel, P. Evolution of charge and spin state of transition metals in the LaMn1-xCoxO3 perovskite series. J. Appl. Phys. 103, 07C907 (2008).

69. Yamaoka, H. et al. Bulk electronic properties of FeSil-xGex investigated by high-resolution X-ray spectroscopies. Phys. Rev. B 77, 115201 (2008).

70. Burnham, K. P. \& Anderson, D. R. Model Selection and Multimodel Inference: A Practical Information-Theoretic Approach 2nd edn (Springer, 2002), ISBN 0-387-95364-7.

\section{Acknowledgements}

This project was supported by the X-ray Free-Electron Laser Priority Strategy Program of MEXT, Japan (to SA), the Swedish Research Council, the Knut and Alice Wallenberg Foundation, the Crafoord Foundation, the Swedish Energy Administration, the Science Faculty at Lund University (MAXIV and ESS initiative grant), the Danish National Research Foundation Center for Molecular Movies, DANSCATT, The Carlsberg Foundation, the Lundbeck Foundation, the European Research Council (ERC-AdvGVISCHEM-226136 to V.S. and ERC-StG-259709 to G.V.), by the European XFEL (CB, WG, AG), by the German Research Foundation (DFG, SFB925, TP A4), by the Centre of Ultrafast Imaging (CUI), the Hungarian Academy of Sciences via the Lendület (Momentum) Program (G.V.), and the Bolyai Fellowship (Z.N.). The XFEL experiments were performed at the BL3 of SACLA with the approval of JASRI (Proposal No. 2012A8049). Use of the Center for Nanoscale Materials was supported by the US 
Department of Energy, Office of Science, Office of Basic Energy Sciences, under Contract No. DE-AC02-06CH11357.

\section{Additional information}

Supplementary Information accompanies this paper at http://www.nature.com/ naturecommunications

Competing financial interests: There are no competing financial interests.

Reprints and permission information is available online at http://npg.nature.com/ reprintsandpermissions/
How to cite this article: Canton, S. E. et al. Visualizing the non-equilibrium dynamics of photoinduced intramolecular electron transfer with femtosecond X-ray pulses. Nat. Commun. 6:6359 doi: 10.1038/ncomms7359 (2015).

(c) (i) This work is licensed under a Creative Commons Attribution 4.0 International License. The images or other third party material in this article are included in the article's Creative Commons license, unless indicated otherwise in the credit line; if the material is not included under the Creative Commons license, users will need to obtain permission from the license holder to reproduce the material. To view a copy of this license, visit http://creativecommons.org/licenses/by/4.0/ 